\title{
Adaptación, validez y confiabilidad de la escala de detección de sexismo (DSE) en estudiantes del nivel secundaria
}

Adaptation, validity and reliability of the sexism detection scale

(DSE) in secondary school students

Adaptação, validade e confiabilidade da escala de detecção de sexismo

(DSE) em estudantes do ensino médio

\author{
GALIA SUSANA LESCANO LÓPEZ ${ }^{1}$ \\ DORA LOURDES PONCE YACTAYO ${ }^{2}$
}

\begin{abstract}
RESUMEN
La investigación tuvo como objetivo realizar la adaptación, la validez y confiabilidad de la escala de detección de sexismo en estudiantes del nivel secundaria (DSE). La muestra estuvo constituida por 561 estudiantes del nivel secundaria de cuatro instituciones educativas de gestión pública del distrito de Comas. Mediante el análisis factorial exploratorio y confirmatorio, se contrastó la idoneidad de la escala con dos factores (sexismo hostil y sexismo benévolo), asimismo los resultados evidencian un alto nivel de consistencia interna ( $\alpha=$ $0.911)$ y de varianza explicada (41\%).
\end{abstract}

Palabras clave: Sexismo; escala; validez; confiabilidad; estudiantes.

\begin{abstract}
The research aimed to carry out the adaptation, validity and reliability of the sexism detection scale in secondary school students (DSS). The sample consisted of 561 secondary school students from four public management educational institutions in the Comas district. Using the exploratory and confirmatory factor analysis, the suitability of the scale was contrasted with two factors (hostile sexism and benevolent sexism). Likewise, the results show a high level of internal consistency $(\alpha=0.911)$ and of explained variance $(41 \%)$.
\end{abstract}

Keywords: Sexism; scale; validity; reliability; students. 


\section{RESUMO}

A pesquisa objetivou realizar a adaptação, validade e confiabilidade da escala de detecção de sexismo em estudantes do ensino médio (EED). A amostra foi composta por 561 estudantes do ensino médio de quatro instituições de ensino da gestão pública do distrito de Comas. Utilizando a análise fatorial exploratória e confirmatória, a adequação da escala foi contrastada com dois fatores (sexismo hostil e sexismo benevolente). Da mesma forma, os resultados mostram um alto nível de consistência interna $(\alpha=0,911)$ e de variância explicada $(41 \%)$.

Palavras-chave: Sexismo; escala; validade; confiabilidade; alunos.

\section{INTRODUCCIÓN}

Se pretende estudiar el sistema de creencias sexistas en adolescentes para su prevención e intervención en materia de violencia de género contra la mujer. El sexismo y la violencia no son un infortunio biológico, sino más bien, una consecuencia cultural, donde se reproduce un modelo social generacionalmente heredado, que se basa en la dominación y sometimiento. (Díaz, 2016). Actualmente se presentan estudios sobre sexismo en el ámbito educativo (Heilman, 2015; Kurtz-Costes, Copping, Oowley \& Kinlaw, 2014) para conocer la persistencia de los estereotipos de género en estudiantes desde la etapa de la pubertad, donde se inician las relaciones de pareja, para así contribuir a neutralizar los posibles sesgos de corte sexista (Delpino \& Eresta, 2013). Los estereotipos de género hacen referencia a lo que se entiende por sexo y género. Así tenemos, que el sexo hace referencia a las diferencias biológicas entre mujeres y hombres, mientras que el género lo hace en base a las características sociales, que según Colás \& Villaciervos (2007), son la "representación cultural, ideas, prejuicios, valores, interpretaciones, normas, deberes, mandatos y prohibiciones sobre la vida de las mujeres y de los hombres" (p. 37), roles característicos de uno y otro sexo en determinada sociedad (de Lemus, Castillo, Moya, Padilla \& Ryan, 2008). En este sentido los estereotipos de género, desde el punto de vista social, son el fiel reflejo de una cultura y una historia, por tanto, juegan un papel fundamental en la transmisión y perpetuación de los estereotipos de género tradicionales (Vázquez-Cupeiro, 2015) modelando la identidad de género. De este modo, la familia y la escuela son los principales espacios de socialización en los que se aprenden e interiorizan los roles masculinos y femeninos (Perry \& Pauletti, 2011).

En este sentido la escala que se presenta nos permitirá identificar los factores de riesgo y protección en relación a los planteamientos sexistas de los adolescentes referidos a los roles que vienen presentando los hombres y mujeres, para así poder orientar su sistema de creencias personales dentro del contexto educativo en donde la escuela refuerza estereotipos de género que se generan en la familia influenciando en el autoconcepto y logro académico (Igbo, Onu, \& Obiyo, 2015).

El sexismo basado en creencias y actitudes de autoridad y dominio del varón sobre la mujer (Garaigordobil \& Aliri, 2013), considerado como la atribución estereotipada de rasgos a una persona por el simple hecho de ser un hombre o una mujer" (Recio, Cuadrado \& Ramos, 2007, p.522), una de sus graves consecuencias es, la violencia contra las mujeres 
(García, Palacios, Torrico \& Navarro, 2009; Herrera, Expósito \& Moya, 2012; Yamawaki, Ostenson \& Brown, 2009).

En la actualidad los estudios han evidenciado dos conceptualizaciones sobre sexismo: un sexismo tradicional y otro moderno. El sexismo tradicional, definido como "una actitud prejuiciosa o conducta discriminatoria basada en la supuesta inferioridad de las mujeres como grupo" (Cameron, 1997), basado en tres creencias: a) El paternalismo dominador, que sostiene que las mujeres son seres más débiles e inferiores a los hombres; b) La diferenciación de género competitiva; c) La hostilidad heterosexual, que mantiene que las mujeres son peligrosas y manipuladoras de hombres.

El nuevo sexismo desarrollado por Glick \& Fiske $(1996,2001)$ quienes proponen que las relaciones estructurales entre hombres y mujeres, generan actitudes ambivalentes, es así como plantean una estructura bidimensional con cargas afectivas positivas y negativas, opuestas entre sí, dando lugar a dos tipos de sexismo vinculados: sexismo hostil y sexismo benevolente.

Cuando se habla de sexismo hostil, se puede decir que hace referencia al sexismo tradicional, el que legitima el control de los hombres sobre las mujeres, ubicándolas como grupo inferior y subordinado y manifestándose frente a las mujeres que no son estereotípicamente femeninas (Glick \& Fiske, 2001).

En tanto sexismo benevolente, el hombre las idealiza desde la perspectiva tradicional, las limita a los roles tradicionalmente femeninos, y las considera dignas de alabanzas y que las cuiden y protejan (Glick \& Fiske, 1996).

Los autores de esta teoría elaboran un instrumento para medir ambos tipos de sexismo, el Ambivalent Sexism Inventory (ASI) de Glick y Fiske (1996), adaptado y validado en España por Expósito, Moya \& Glick (1998), instrumento que se requiere adaptar y validar en nuestra población de estudio.

\section{METODOLOGÍA}

En el estudio participaron 561 estudiantes del tercero, cuarto y quinto año del nivel secundaria de las instituciones educativas: Estados Unidos, Simón Bolívar, José Carlos Mariátegui y Túpac Amaru pertenecientes al distrito de Comas. La edad promedio fue de 15,34 años (DS =1.26). Del total de participantes, 304 son mujeres y 257 son hombres. La diferencia entre la edad promedio de hombres y mujeres no resultó estadísticamente significativa ( $\mathrm{t}$ $=-0.63, \mathrm{p}=0,958)$. El conjunto inicial de ítems estaba formado por 26 ítems agrupados teóricamente en dos subescalas: Sexismo hostil (16 ítems) y Sexismo benévolo (10 ítems). Los ítems presentan un formato de respuesta politómico con 6 alternativas de respuesta. El sistema de cuantificación asigna valores enteros consecutivos en un rango desde 1 punto (Totalmente en desacuerdo) hasta 6 puntos (Totalmente de acuerdo). Se solicitó las autorizaciones a los directores designados de las cuatro instituciones educativas para la aplicación de la escala de detección de sexismo, el cuestionario fue anónimo, el muestreo fue probabilístico. La confiabilidad de la escala fue analizada mediante el método de consistencia interna, resultado todos los coeficientes de confiabilidad aceptable $(\alpha=0,911)$ 
en la escala general y en las subescalas también resultaron aceptables (sexismo hostil: $\alpha=$ 0,880 y sexismo benévolo: $\alpha=0,849$ ). Se realizó el análisis factorial exploratorio donde la prueba de KMO y Barlett, según la Medida Kaiser-Meyer-Olkin de adecuación de muestreo el modelo factorial es adecuado $(\mathrm{KMO}>0.60)$ y según la prueba de esfericidad de Barlett también el modelo factorial es adecuado $(\mathrm{p}=0.000<0.05)($ Pérez, 2013) y según la varianza total explicada los dos componentes principales tienen varianza (autovalor) mayor que 1, en consecuencia el número de factores elegidos son 2 (sexismo hostil: 5, 9, 10,12, 14 , $16,18,19,20,22,23,24,25,26)$ y sexismo benévolo: $1,2,3,4,6,7,8,11,13,15,17,21$ ). (Tabla 2).

\section{RESULTADOS Y DISCUSIÓN}

Luego de la adaptación de los ítems de la escala de detección del sexismo al entorno peruano, se encontró que los 25 ítems tienen alta correlacion con el puntaje total donde se observa que los índices de discriminación (I.D) son en la mayoría aceptables (entre 0,437 y 0.626). (Tabla 1)

\section{Tabla 1}

Análisis de items de la escala de detección de sexismo

\begin{tabular}{ccccc}
\hline Ítems & Media & varianza & I.D. & Alfa de Cronbach \\
\hline 1 & 65.1800 & 432.598 & 0.196 & 0.914 \\
2 & 65.8770 & 411.062 & 0.486 & 0.909 \\
3 & 65.7861 & 412.876 & 0.483 & 0.909 \\
4 & 66.6881 & 415.161 & 0.496 & 0.908 \\
5 & 66.0606 & 410.514 & 0.558 & 0.907 \\
6 & 66.8324 & 415.100 & 0.531 & 0.908 \\
7 & 65.7184 & 411.249 & 0.526 & 0.908 \\
8 & 65.6845 & 406.316 & 0.523 & 0.908 \\
9 & 66.1052 & 411.884 & 0.568 & 0.907 \\
10 & 66.0071 & 405.064 & 0.626 & 0.906 \\
11 & 65.7914 & 416.080 & 0.461 & 0.909 \\
12 & 66.1283 & 412.558 & 0.503 & 0.908 \\
13 & 67.0000 & 421.643 & 0.455 & 0.909 \\
14 & 67.2709 & 424.451 & 0.455 & 0.909 \\
15 & 67.2086 & 419.605 & 0.496 & 0.909 \\
16 & 66.4207 & 417.669 & 0.437 & 0.910 \\
17 & 66.0053 & 403.412 & 0.611 & 0.906 \\
18 & 66.8610 & 413.673 & 0.541 & 0.908 \\
19 & 66.7611 & 415.761 & 0.559 & 0.907 \\
20 & 66.8396 & 415.981 & 0.577 & 0.907 \\
21 & 66.8984 & 414.859 & 0.563 & 0.907 \\
22 & 66.9109 & 420.646 & 0.479 & 0.909 \\
23 & 66.7843 & 411.894 & 0.572 & 0.907 \\
24 & 66.7522 & 413.458 & 0.546 & 0.908 \\
25 & 66.9216 & 416.569 & 0.519 & 0.908 \\
26 & 65.6203 & 406.347 & 0.519 & 0.908 \\
\hline & & & & \\
\hline
\end{tabular}


También se evaluó el valor del determinante de la matriz de correlaciones, la cual tiene como finalidad conocer si los ítems están linealmente relacionados, donde el valor tiene que aproximarse a cero (Determinante $=0,0000269$ ).

Tabla 2

Componentes principales

\begin{tabular}{ccc}
\hline \multirow{2}{*}{ Ítems } & \multicolumn{2}{c}{ Componente } \\
\hline 1 & -0.239 & 0.611 \\
2 & 0.335 & 0.425 \\
3 & 0.119 & 0.664 \\
4 & 0.308 & 0.477 \\
5 & 0.497 & 0.207 \\
6 & 0.228 & 0.654 \\
7 & 0.355 & 0.479 \\
8 & 0.170 & 0.670 \\
9 & 0.540 & 0.154 \\
10 & 0.717 & 0.013 \\
11 & 0.348 & 0.466 \\
12 & 0.531 & 0.139 \\
13 & 0.232 & 0.660 \\
14 & 0.574 & 0.351 \\
15 & 0.381 & 0.583 \\
16 & 0.765 & 0.043 \\
17 & 0.170 & 0.565 \\
18 & 0.684 & 0.150 \\
19 & 0.640 & 0.230 \\
20 & 0.593 & 0.265 \\
21 & 0.205 & 0.592 \\
22 & 0.489 & 0.253 \\
23 & 0.641 & 0.220 \\
24 & 0.564 & 0.270 \\
25 & 0.597 & 0.187 \\
26 & 0.446 & 0.350 \\
\hline & & \\
\hline & & 0.59 \\
\hline
\end{tabular}

Mediante el análisis factorial exploratorio, según Chión \& Vincent (2016) el número de factores se determina por medio de ACP (análisis de componentes principales) donde se encuentran los autovalores, en los resultados se encontró dos factores que explican la varianza común de los ítems, según el criterio de Kaiser se seleccionó aquellos componentes cuyos autovalores (varianza explicada) sea mayor que la unidad (Tabla 2).

En la figura 1, se observa el gráfico de sedimentación, Catell (1966 citado en Véliz, 2017, p. 37) indicó que "el gráfico de sedimentación es apropiado cuando los factores son extraídos por el método de componentes principales" la cual nos permite visualizar el número óptimo de componentes (factores), donde se aprecia de izquierda a derecha una evidente inflexión a partir del tercer autovalor, por lo que se toma la decisión de extraer solo dos factores. 


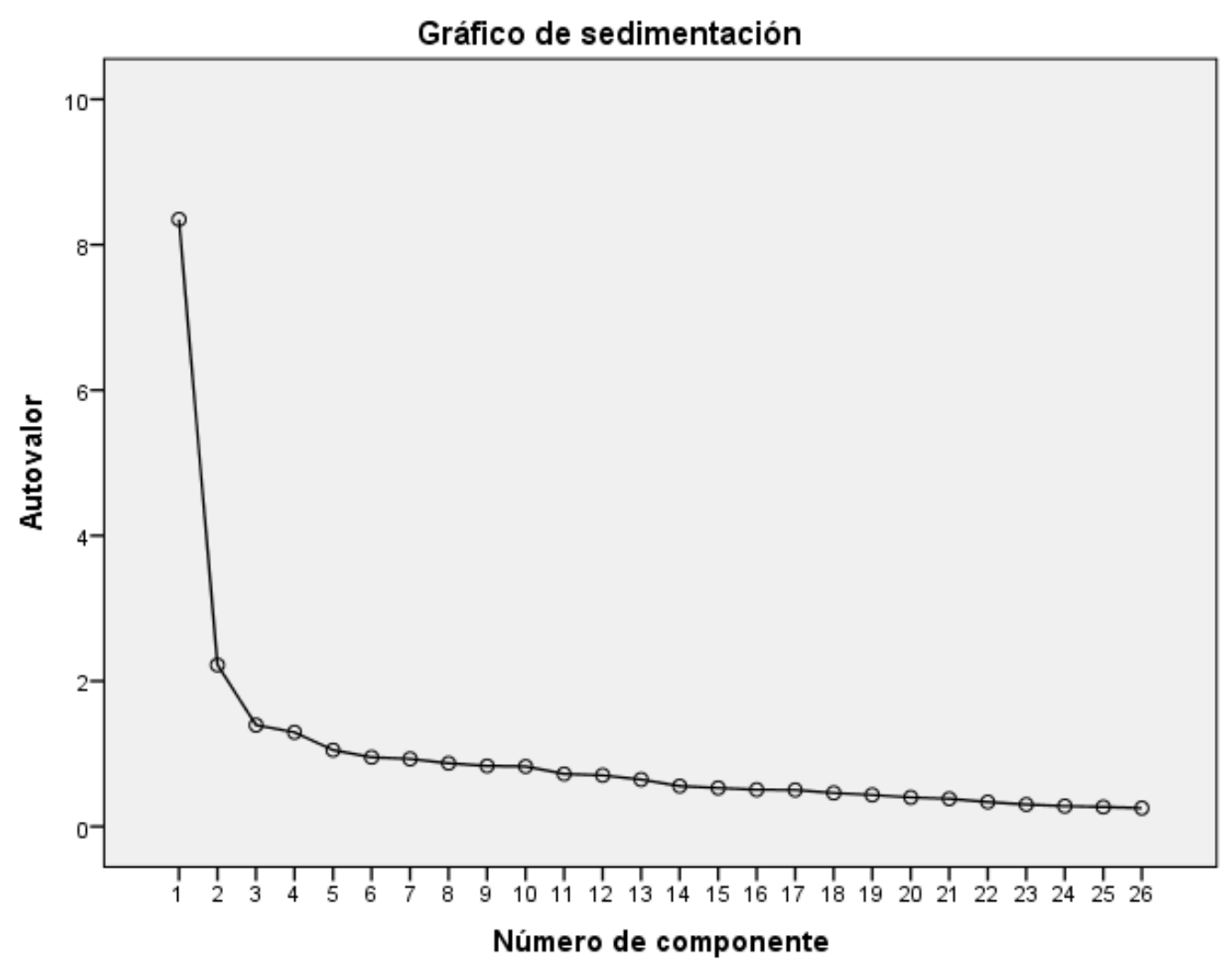

Figura 1. Gráfico de sedimentaciones de la Escala de Detección de Sexismo en estudiantes de secundaria.

\section{Análisis factorial confirmatorio}

Se realizó un análisis factorial confirmatorio utilizando el análisis factorial confirmatorio utilizando la extensión AMOS de SPSS, donde se demostró el modelo con los dos factores (sexismo hostil y sexismo benévolo) las cuales serien las variables latentes, al respecto Sastre (2018) indicó que "las variables latentes son las que no se pueden observar directamente, son constructos hipotéticos" (p. 12) y los ítems son cargas factoriales en cada factor llamada también dimensión en la que los dos factores muestran un buen ajuste del modelo según el análisis factorial confirmatorio.

\section{CONCLUSIONES}

Los resultados de la investigación indican que la escala de detección de sexismo en estudiantes de secundaria presenta características psicométricas para su utilización en el Perú, donde se obtienen resultados similares en la investigación realizada por Recio, Cuadrado y Ramos (2007) en España y la investigación realizada por Lemus, Castillo, Moya, Padilla \& Ryan (2008) en estudiantes del nivel secundaria de Granada, España.

La detección del sexismo en estudiantes del nivel secundaria permite tomar medidas de prevención de la violencia de género de los estudiantes.

La escala de detección de sexismo es confiable en los dos factores encontrados, confirmando los resultados de la investigación de Recio, Cuadrado \& Ramos (2007) en España. 


\section{REFERENCIAS}

Cameron, C., (1977). Sex-role attitudes. En Attitudes and opinions. Prentice Hall, pp. 339359.

Colás, P., \& Villaciervos, P. (2007). La interiorización de los estereotipos de género en jóvenes y adolescentes. Revista de Investigación Educativa, 1(25), 35-58. http:// revistas.um.es/rie/article/view/96421/92631

Chión, S. \& Vincent, C. (2016). Analítica de datos para la modelación estructural. Pearson.

De Lemus, S., Castillo, M., Moya, M., Padilla, J. L., \& Ryan, E. (2008). Elaboración y validación del inventario de sexismo ambivalente para adolescentes. International Journal of Clinical and Health Psychology, 8(2), 537-562. https://www.redalyc.org/ pdf/337/33712001013.pdf

Delpino, M., \& Eresta, M. (2013). Relaciones afectivas y sexualidad en la adolescencia. Ministerio de Sanidad, Servicios Sociales e Igualdad.

Díaz, M. (2016). La prevención de la violencia de género entre adolescentes. Educació Social. Revista d'Intervenció Socioeducativa, 63, 11-30.

Expósito, F., Moya, M. C., \& Glick, P. (1998). Sexismo ambivalente: medición y correlatos. Revista de Psicología Social, 13(2), 159-169. https://doi. org/10.1174/021347498760350641

Garaigordobil, M. \& Aliri, J. (2013). Relaciones del sexismo con justificación de la violencia, y con otras formas de prejuicio como la dominancia social y el autoritarismo. Estudios de Psicología, 34(2), 127-139. http://www.tandfonline.com/loi/redp20

García, P., Palacios M.S., Torrico, E., \& Navarro, Y. (2009). El sexismo ambivalente ¿un predictor de maltrato? Asociación Latinoamericana de Psicología jurídica y Forense. http://psicologiajuridica.org/psj210.html

Glick, P., \& Fiske, S. T. (1996). The Ambivalent Sexism Inventory: differentiating hostile and benevolent sexism. Journal of Personality and Social Psychology, 70(3), 491512.

Glick, P. \& Fiske, S. (2001). Ambivalent sexism. Advances in Experimental. Social Psychology, 33, 115-188

Herrera, M. C., Expósito, F., Moya, M. y Houston, D. M. (2012). “Having it all”: Women’s perception of impact of female promotion on threat of domestic violence. The Spanish Journal of Psychology, 15, 670-679.

Heilman, M. (2015). Gender stereotypes: Impediments to women's career progress. En I. Welpe, P. Brosi, L. Ritzehöfer, \& T. Schwarzmüller (Eds.), Auswhal von Männern und Frauen als Führungskräfte. Perspektiven aus Wirtschaft, Wissenschaft, Medien und Politik. pp. 73-84.

Igbo, J.N., Onu, V.C., \& Obiyo, N.O. (2015). Impact of gender stereotype on secondary school students`self-concept and academic achievement. Sage Open, 5(1), 1-10. 
Kurtz-Costes, B., Copping, K.E., Rowley, S.J., \& Kinlaw, C.R. (2014). Gender and age differences in awareness and endorsement of gender stereotypes about academic abilities. European Journal of Psychology of education, 29(4), 603-618.

Lemus, S., Castillo, M., Moya, M., Padilla, J., \& Ryan, E. Elaboración y validación del Inventario de Sexismo Ambivalente para Adolescentes (2008). International Journal of Clinical and Health Psychology, 8 (2), 537-562.

Palacios, M., Torrico, E., Millán, M., Pérez, M., Puertas, S., Moya, M., \& López, J. (2005). Violencia contra las mujeres y sexismo ambivalente. En J. Sobral, G. Serrano y J. Regueiro (Comps.), Psicología jurídica, de la violencia y de géneropp. Biblioteca Nueva. pp. 345-357.

Pérez, C. (2013). Análisis multivariante de datos. Garceta.

Perry, D., \& Pauletti, R. (2011). Gender and adolescent development. Journal of Research on Adolescence, 21(1), 61-74.

Recio, P., Cuadrado, I., \& Ramos, E. (2007). Propiedades psicométricas de la Escala de detección de sexismo en adolescentes (DSA). Psicothema, 19 (3), 522-528.

Sastre, P. (2018). Modelo de ecuaciones estructurales. Nociones básicas. Editorial Académica Española.

Vázquez-Cupeiro, S. (2015). Ciencia, estereotipos y género. Una revisión de los marcos explicativos. Convergencia: Revista de ciencias sociales, 22(68), 177-202.

Véliz, C. (2017). Análisis multivariante. Métodos estadísticos multivariantes para la investigación. Cengaje-Learning.

Yamawaki, N., Ostenson, J., \& Brown, C. R. 2009. The functions of gender role traditionality, ambivalent sexism, injury, an frecuency of assault on domestic violence perception. Violence Against Women, 15, 1126 -1142. 